\title{
Effects of a New Antiallergic Drug, VUF-K-8788, on Infiltration of Lung Parenchyma by Eosinophils in Guinea Pigs and Eosinophil-Adhesion to Human Umbilical Vein Endothelial Cells (HUVEC)
}

\author{
Toshiaki Takizawa, ${ }^{*, a, b}$ Chie Watanabe, ${ }^{c}$ Ikuo SAIKI, ${ }^{c}$ Yasushi WadA, ${ }^{b}$ Tsutomu Tohma, ${ }^{b}$ and \\ Hiroichi NAGAI ${ }^{a}$ \\ Department of Pharmacology, Gifu Pharmaceutical University, ${ }^{a}$ Gifu 502-8585, Japan, Tokyo Research Laboratories, \\ Kowa Company Ltd., b Higashimurayama, Tokyo 189-0022, Japan, and Research Institute for Wakan-yaku, Toyama \\ Medical and Pharmaceutical University, ${ }^{c}$ Toyama, Japan. $\quad$ Received March 29, 2001; accepted June 19, 2001
}

\begin{abstract}
Airway inflammation and reversible airway obstruction are hallmarks of bronchial asthma. In this study, we investigated the effects of a new antiallergic drug, 7-\{3-[4-(2-quinolinylmethyl)-1-piperazinyl]-propoxy\}-2,3-dihydro-4H-1,4-benzothiazin-3-one (VUF-K-8788), on histopathological changes in lung parenchyma of guinea pigs during late-phase asthmatic reaction (LAR), and on eosinophil-adhesion to human umbilical vein endothelial cells (HUVEC).

Repeated exposure to ovalbumin of sensitized guinea pigs induced inflammatory phenomena such as hyperplasia of airway epithelial cells, perivascular edema and infiltration of lung parenchyma by eosinophils. VUF-K8788 inhibited these histopathological phenomena at $10 \mathrm{mg} / \mathrm{kg}$ p.o. Moreover, the eosinophil-adherence to HUVEC was inhibited by VUF-K-8788 at the concentration of $10-30 \mu_{\mathrm{M}}$. In conclusion, this inhibitory effect of VUF-K-8788 on eosinophil-adherence might contribute to the prevention of LAR and infiltration by eosinophils in the experimental asthmatic model in guinea pigs.
\end{abstract}

Key words VUF-K-8788; asthma; eosinophil; human umbilical vein endothelial (HUVE) cell; guinea pig

Allergen provocation induces immediate- and late-phase reactions in allergic disorders, such as bronchial asthma, ${ }^{1)}$ atopic dermatitis ${ }^{2)}$ or allergic rhinitis. ${ }^{3)}$ Pepys and Hutchcroft $\left.^{4}\right)$ reported that immediate- and late-phase asthmatic responses were observed $15-30 \mathrm{~min}$ and $6-12 \mathrm{~h}$ after allergen provocation, respectively. Hargreave et al. ${ }^{1,5)}$ and Cartier et $\left.a{ }^{6}{ }^{6}\right)$ reported that late-phase asthmatic reactions (LAR) were observed up to 2 or $3 \mathrm{~d}$ after allergen provocation and were accompanied by airway hyperresponsiveness to a wide variety of stimuli including histamine and methacholine. The development of LAR is associated with the influx and activation of eosinophils. ${ }^{\text {) }}$

A new model for biphasic asthmatic response in guinea pigs was reported by Takeda $e t$ al. ${ }^{8)}$ In this model, LAR was caused by repeated airway inflammation with eosinophilia and chemical mediator release including histamine, thromboxane $\mathrm{A}_{2}$ and leukotriene $\mathrm{D}_{4}$. VUF-K-8788, 7-\{3-[4-(2quinolinylmethyl)-1-piperazinyl]-propoxy $\}$-2,3-dihydro- $4 H$ 1,4-benzothiazin-3-one, is a newly synthesized anti-allergic drug, which antagonized histamine $\mathrm{H}_{1}$ receptor and inhibited the biphasic asthmatic reaction in guinea pigs. $\left.{ }^{9}, 10\right)$

One of the mechanisms which is important in the process of infiltration by eosinophil involves the interaction of adhesion molecules on the surface of the circulating cells and the vascular endothelium. ${ }^{11,12}$ Bochner et al. reported that eosinophils express functional counter-ligands for the inducible adhesion molecules on the endothelial surface including the vascular cell adhesion molecule-1 (VCAM-1, CD106) and the intercellular adhesion molecule-1 (ICAM-1, CD54). ${ }^{13)}$

In our previous experiment, $\left.{ }^{9}, 10\right)$ VUF-K-8788 (0.3$10 \mathrm{mg} / \mathrm{kg}$ ) inhibited immediate- and late-phase allergic reactions, airway hyperresponsiveness and eosinophils in bronchoalveolar fluids, while terfenadine $(100 \mathrm{mg} / \mathrm{kg})$ inhibited only the immediate reaction. In this study, we investigated the effect of VUF-K-8788 on histopathological changes in lung parenchyma in guinea pigs during LAR, eosinophil-adhesion to human umbilical vein endothelial cells (HUVEC), and expression of adhesion molecules on HUVEC.

\section{MATERIALS AND METHODS}

Animals Male Hartley guinea pigs aged 4 weeks (body weight: $250-300 \mathrm{~g}$ ) were purchased from Japan SLC Inc. (Hamamatsu, Japan). The animals were fed standard laboratory diet and water ad libitum in an air-conditioned room at $23 \pm 3{ }^{\circ} \mathrm{C}$ and relative humidity of $55 \pm 15 \%$.

The experiments were carried out in accordance with the Guidelines for the Care and Use of Laboratory Animals of Gifu Pharmaceutical University.

Materials HUVEC (Seikagaku Kogyo Inc., Tokyo, Japan), HUVE cell culture cedium (Nissui Seiyaku Inc., Tokyo, Japan), CD16 Ab (MG 38, mouse anti-human $\operatorname{IgG}_{1}$, Nichirei, Tokyo, Japan), sheep anti-mouse $\operatorname{IgG}_{1}$ coated beads (Dynal As, Oslo, Norway), bovine endothelial mitogen (Biomedical Technologies Inc., Stoughton, MA, U.S.A.), recombinant human TNF- $\alpha$ (Peptide Tech EC LTD, London, England), Diffquick Solution (Kokusai-Shiyaku Inc., Kobe, Japan), anti-human VCAM-1, anti-human CD54 (ICAM-1), and FITC-labeled rabbit anti-mouse $\operatorname{IgG}_{1}$ conjugate (Endogen Inc., MA, U.S.A.) were purchased. VUF-K-8788 was synthesized in Kowa Tokyo Laboratories (Tokyo). Ovalbu$\min (\mathrm{OA})$ and other chemicals were purchased from SIGMA Inc. (MO, U.S.A.).

Induction of LAR and Histopathological Analysis Induction of LAR was carried out as previously described. ${ }^{10)}$ In brief, male Hartley guinea pigs were actively sensitized twice with OA $(0.5 \mathrm{mg} /$ body i.p. on day 1 and $1 \mathrm{mg} /$ body i.p. on day 3). Actively sensitized guinea pigs were repeatedly exposed to aerosolized antigen. One-minute inhalations of 0.1 , 
$0.2,0.4,0.5,1$ and $1 \%$ OA solution were administered on days $23,25,28,32,37$ and 39 , respectively, using an ultrasonic nebulizer (TUR-3200, Nihon Kohden, Tokyo, Japan). VUF-K-8788 or terfenadine was administered orally $1 \mathrm{~h}$ before the inhalations. Four hours after the last inhalation, when LAR had been induced, the animals were sacrificed. The lungs including bronchi were dissected and were fixed in $10 \%$ neutral buffered formalin. Sections $3 \mu \mathrm{m}$ thick were stained with hematoxylin-eosin or Hansel's solution. The histopathological changes, such as hyperplasia of airway epithelium and perivascular edema, were scored as follows by the method of Blyth et al. ${ }^{14)}$ : 0: none, 100: occasional, 200: small, 300: moderate, 400: heavy, 500: massive. Each score was evaluated by comparing with typical histological standards which were taken in preliminary experiments. Eosinophils that infiltrated to lung parenchyma were counted by the method of Sagara et al. ${ }^{15)}$ For cell counting, each slide glass was coded and read in a "blind" fashion. The regions were chosen at random in the sections.

Cell Adhesion Assay Cell Culture: HUVEC were cultured in HUVE cell culture medium at $37^{\circ} \mathrm{C}$ in a $5 \% \mathrm{CO}_{2}$ incubator. HUVEC were subcultured every week and were used within the first 6 passages.

Eosinophil Purification: Blood from asthmatic volunteers $(40 \mathrm{ml})$ was mixed with $10 \mathrm{ml}$ of $40 \%$ dextrin-saline solution and kept at room temperature for $1 \mathrm{~h}$. Supernatant was layered over lymphocyte separation medium and centrifuged at $20^{\circ} \mathrm{C}, 200 \times \boldsymbol{g}$, for $7 \mathrm{~min}$. Polymorphonuclear cells (PMN) were washed twice with PBS and resuspended in RPMI 1640 supplemented with 5\% fetal bovine serum (FBS) and $4 \mathrm{~mm}$ EDTA. Immunomagnetic negative selection, using CD16 Ab and sheep anti-mouse $\mathrm{IgG}_{1}$ coated beads, was executed to purify eosinophils from PMN. The purity of eosinophils was $80.6 \%$.

Cell Adhesion Assay: The subcultured HUVEC were collected and resuspended in D-MEM/F12 medium supplemented with $10 \%$ FBS, bovine endothelial mitogen $100 \mu \mathrm{g} / \mathrm{ml}$ and recombinant human TNF- $\alpha$. They were seeded on 96 -well plates at $1 \times 10^{5}$ cells in $100 \mu \mathrm{l}$ of medium, and preincubated in a $\mathrm{CO}_{2}$-incubator. After $1 \mathrm{~h}$ incubation, VUF-K-8788 or terfenadine was added to confluent HUVEC in $100 \mu \mathrm{l}$ of HUVE cell medium. Following $24 \mathrm{~h}$ incubation, eosinophils $\left(1 \times 10^{5}\right.$ cells) were added to HUVEC and co-incubated for $30 \mathrm{~min}$. The un-adhered eosinophils were then washed out with PBS, and the eosinophils adhering to HUVEC were stained with Diffquick Solution and counted under a microscope at a magnification of $\times 400$.

Flowcytometry Subcultured HUVEC were seeded on 6well plates at $1 \times 10^{5}$ cells in $2 \mathrm{ml}$ of HUVEC medium and cultured for $3 \mathrm{~d}$. HUVEC monolayers were rinsed with $1 \mathrm{ml}$ of warmed PBS, and TNF- $\alpha$ and test compounds in $2 \mathrm{ml}$ of D-MEM/F12 medium containing $10 \%$ FBS and $100 \mu \mathrm{g} / \mathrm{ml}$ endothelial mitogen were added. After $24 \mathrm{~h}$ incubation, HUVEC were treated with $20 \%$ trypsin-EDTA and collected. Single cell suspensions were centrifuged $(2000 \times \boldsymbol{g}$, $30 \mathrm{~s}$ ) and resuspended in PBS.

Cells were incubated in microtubes at $4{ }^{\circ} \mathrm{C}$, first with the primary mAbs (anti-human VCAM-1 or anti-human CD54, $30 \mathrm{~min}$ ) and next with the FITC-labeled rabbit anti-mouse $\mathrm{IgG}_{1}$ conjugate $(30 \mathrm{~min})$. Cells were washed twice by centrifugation after each incubation. The final suspension was made in $500 \mu \mathrm{l}$ of medium containing propidium iodide $(2.5 \mu \mathrm{g} / \mathrm{ml})$. Irrelevant isotype-matched primary Abs served as control. Cell samples were analyzed on FACScan (Becton Dickinson Inc., CA, U.S.A.).

Cytotoxicity for HUVEC and Eosinophils To investigate the cytotoxicity of the test compounds for HUVEC and eosinophils, the crystal violet staining assay and WST-1 cell counting Kit (Dojindo, Kumamoto, Japan) were applied, respectively. ${ }^{16)}$ In brief, HUVEC were preincubated in HUVE cell medium for $2 \mathrm{~h}$ at $37^{\circ} \mathrm{C}$ in an atmosphere of $5 \% \mathrm{CO}_{2}$ and $95 \%$ air. VUF-K-8788 or terfenadine was added to the medium and the cells were incubated for $24 \mathrm{~h}$. After fixation with glutaraldehyde, the wells were washed with water. The HUVEC remaining in the wells were stained with $0.5 \%$ crystal violet and washed with water. Stained crystal violet was extracted with $30 \%$ acetic acid and quantified by spectrophotometry $(\lambda=590 \mathrm{~nm})$.

Eosinophils were preincubated in RPMI 1640 medium containing $10 \% \mathrm{FBS}$ for $2 \mathrm{~h}$ at $37^{\circ} \mathrm{C}$ in an atmosphere of $5 \%$ $\mathrm{CO}_{2}$ and $95 \%$ air. VUF-K-8788 or terfenadine was added to the medium and the cells were incubated for $24 \mathrm{~h}$. After addition of WST-1 solution, eosinophils were incubated for $4 \mathrm{~h}$. The absorbance of the cell suspension was measured by spectrophotometry $(\lambda=450 \mathrm{~nm})$.

Relative cell numbers in control and drug-treated wells were compared based on the absorbance.

Statistics Student's $t$-test or Mann-Whitney's U-test was used to analyze significance of the difference between groups. Differences were accepted as statistically significant at $p$-values $<0.05$.

\section{RESULTS}

Effects on Hyperplasia and Edema Repeated inhalation of OA to actively sensitized guinea pigs induced hyperplasia of airway epithelium, perivascular edema and infiltration of bronchial parenchyma and airways by eosinophils (Figs. $1 b$ and $3 b$ ). No obvious change was observed in normal animals (Figs. 1a and 3a). Effects of VUF-K-8788 on the hyperplasia and the edema were investigated by morphometric method. VUF-K-8788 (10 mg/kg, Figs. 1c and 2) and terfenadine $(100 \mathrm{mg} / \mathrm{kg}$, Figs. 1d and 2) inhibited the hyperplasia of airway epithelium and perivascular edema significantly.

Effects on Eosinophils Infiltration by eosinophils was evaluated at $4 \mathrm{~h}$ after OA challenge (Figs. 3 and 4). Infiltrating eosinophil counts were $0.9 \pm 0.4$ cells $/ \mathrm{mm}^{2}$ in normal animals and $16.5 \pm 4.4$ cells $/ \mathrm{mm}^{2}$ in control animals (Figs. 3a and $3 \mathrm{~b}$ ). VUF-K-8788 obviously inhibited the infiltration by eosinophils (Figs. 3c and 4), while terfenadine failed to do so (Figs. 3d and 4). There was a significant difference between the effects of VUF-K-8788 and terfenadine on this infiltration.

Eosinophil-Adhesion to HUVEC To investigate the mechanism by which VUF-K-8788 inhibits infiltration of lung parenchyma and airways by eosinophils, we tested whether VUF-K-8788 was capable of inhibiting the eosinophil adhesion to TNF- $\alpha$ stimulated HUVEC. To unstimulated HUVEC, 48 cells/high power field (h.p.f., $\times 400$ ) of eosinophils adhered; after the stimulation with $5 \mathrm{ng} / \mathrm{ml}$ TNF- $\alpha, 95$ cells/h.p.f. of eosinophils adhered. VUF-K-8788 

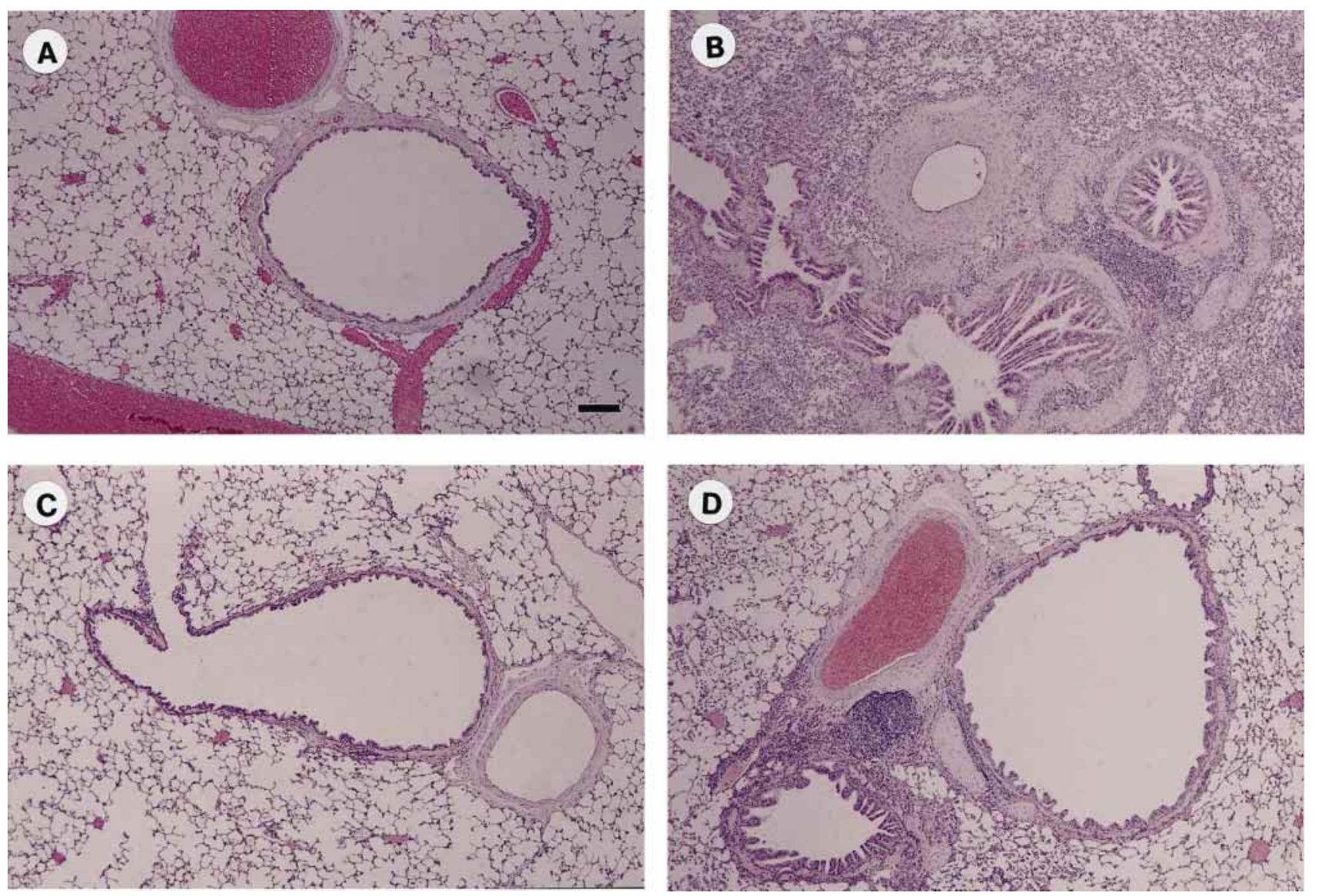

Fig. 1. Hematoxylin and Eosin Staining of Lung Sections from Guinea Pigs

The animals were actively sensitized with OA, and made to inhale OA 6 times. Four hours after the 6th inhalation, lung parenchymas were dissected. The specimens were sectioned and stained with hematoxylin and eosin. A, normal; B, control; C, VUF-K-8788-treated; D, terfenadine-treated. Bar $=200 \mu \mathrm{m}$.
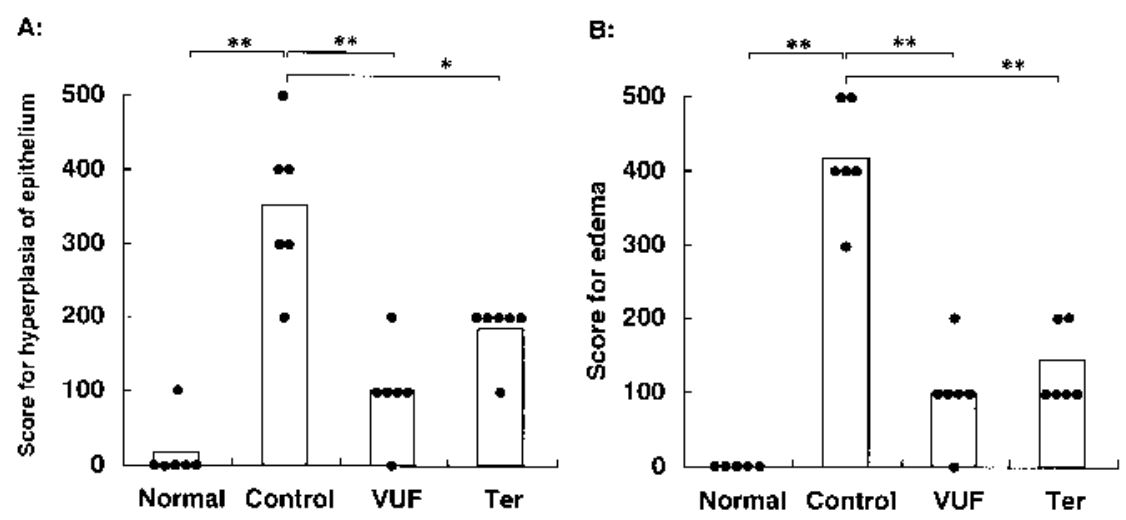

Fig. 2. Effect of VUF-K-8788 (VUF) and Terfenadine (Ter) on Hyperplasia of Epithelium and Perivascular Edema Each column and point represent mean and individual score, respectively. $* p<0.05, * * p<0.01 . n=6$.

inhibited the adhesion of eosinophils to stimulated HUVEC down to the unstimulated level depending on the concentration from 3 to $30 \mu \mathrm{M}$, although terfenadine $(100 \mu \mathrm{M})$ failed to do so (Fig. 5).

Expression of VCAM-1 and ICAM-1 The expression of VCAM-1 and ICAM-1 on HUVEC stimulated with TNF$\alpha$ was also investigated. The mean fluorescence intensity (MFI) of VCAM-1 on unstimulated HUVEC was 82. MFI of VCAM-1 on HUVEC stimulated with TNF- $\alpha$ increased to 138 (Table 1).
VUF-K-8788 $(1-30 \mu \mathrm{M})$ and terfenadine $(100 \mu \mathrm{M})$ did not inhibit the expression of VCAM-1. Dimethylfumarate, as a positive compound, significantly inhibited the expression of VCAM-1.

HUVEC stimulated with TNF- $\alpha$ also expressed ICAM-1. VUF-K-8788 did not affect the expression of ICAM-1, whereas terfenadine and dimethylfumarate inhibited its expression significantly.

Cytotoxicity of the Compounds The cytotoxicity of VUF-K-8788 and terfenadine was examined. As shown in 

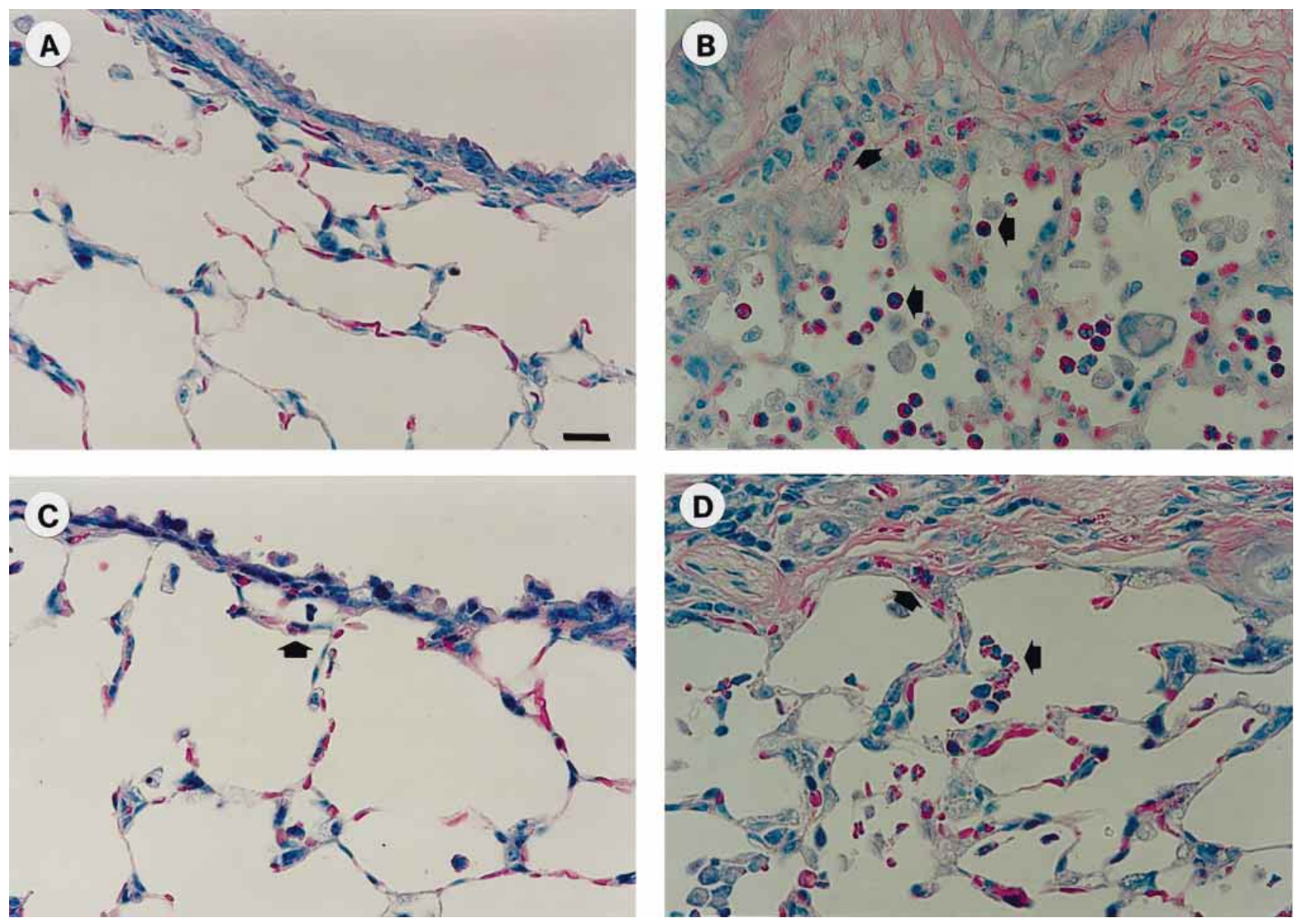

Fig. 3. Hansel's Staining of Lung Sections from Guinea Pigs

The specimens from the animals in Fig. 1 were stained with Hansel's solution. A, normal; B, control; C, VUF-K-8788-treated; D, terfenadine-treated. Note that eosinophils (arrows) infiltrated to lung parenchyma and airway in control animals, which decreased in VUF-K-8788- treated animals. Bar $=20 \mu \mathrm{m}$.

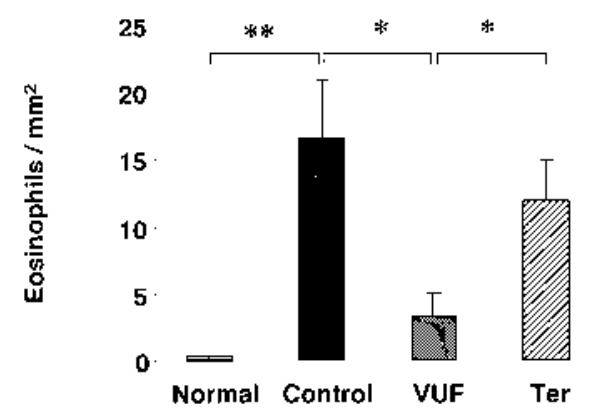

Fig. 4. Effect of VUF-K-8788 (VUF) and Terfenadine (Ter) on Infiltration by Eosinophils

The eosinophils infiltrating lungs were counted. Each value represents mean \pm S.E.M. $* p<0.05, * * p<0.01 . n=6$.

Fig. 6A, VUF-K-8788 did not show cytotoxicity for HUVEC at the concentration of $1-30 \mu \mathrm{M}$; terfenadine $(100 \mu \mathrm{M})$ showed little cytotoxicity. Examination of the cytotoxicity to eosinophils showed no cytotoxicity by either VUF-K-8788 or terfenadine.

\section{DISCUSSION}

Airway inflammation, reversible airway obstruction and airway hyperresponsiveness are the hallmarks of bronchial

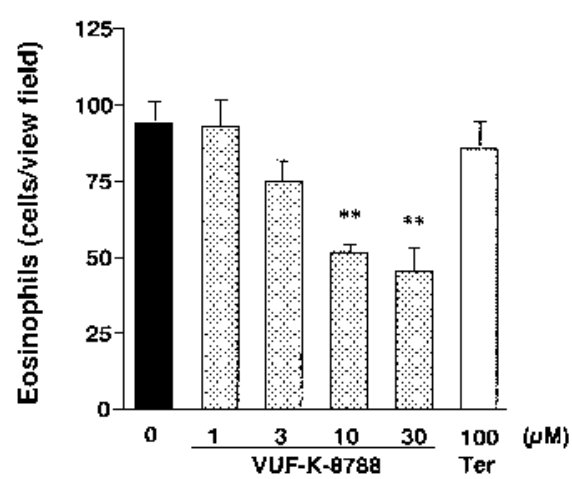

Fig. 5. Effect of VUF-K-8788 on Adhesion of Eosinophils to HUVEC

HUVEC were seeded on 96-well plates at $1 \times 10^{5}$ cells in $100 \mu$ l of HUVE cell medium (D/F12 medium containing $20 \mathrm{ng} / \mathrm{ml} \mathrm{TNF}-\alpha, 10 \%$ FBS and $10 \mu \mathrm{g} / \mathrm{ml}$ bovine endothelial mitogen). After $1 \mathrm{~h}$ incubation, VUF-K-8788 or terfenadine (Ter) was added to confluent HUVEC in $100 \mu 1$ of HUVE cell medium. Following $24 \mathrm{~h}$ incubation, eosinophils $\left(1 \times 10^{5}\right)$ were added to HUVEC and co-incubated for $30 \mathrm{~min}$. Cell adhesion was measured by counting the adhering eosinophils. Each value represents the mean \pm S.E.M. of 5 experiments. $* * p<0.01$.

asthma. ${ }^{17)}$ In the development of bronchial asthma, airway inflammation, characterized by hyperplasia of the airway epithelium, perivascular edema and eosinophil infiltration have been implicated. ${ }^{18)}$

In this study, we investigated effects of VUF-K-8788 on 
the histopathological changes in the lung parenchyma of guinea pigs during the LAR, and the eosinophil-adhesion to HUVEC to elucidate mechanisms by which VUF-K-8788 inhibited the LAR and the infiltration by eosinophils of the bronchoalveolar lavage fluid in guinea pigs.

Repeated exposures to OA of actively sensitized guinea pigs induced both immediate- and late-phase asthmatic reactions one minute and $4 \mathrm{~h}$ after the exposure of antigens, respectively. ${ }^{10)}$ The immediate-phase asthmatic reaction (IAR) was induced by histamine; the histamine antagonist terfenadine inhibited it. During the LAR, various inflammatory phenomena such as hyperplasia of airway epithelial cells, perivascular edema, and infiltration by inflammatory cells, especially eosinophils, were recognized in lung parenchymal specimens. The features were consistent with

Table 1. Effects of VUF-K-8788, Terfenadine and Dimethylfumarate on VCAM-1 and ICAM-1Expression on HUVEC Stimulated with TNF- $\alpha$

\begin{tabular}{ccccc}
\hline \hline & $\begin{array}{c}\text { TNF- } \alpha \\
(20 \mathrm{ng} / \mathrm{ml})\end{array}$ & Compound & Concentration & $\begin{array}{c}\text { MFI } \\
\text { (mean } \pm \text { S.D. })\end{array}$ \\
\hline VCAM-1 & - & - & - & $82 \pm 3$ \\
& + & - & - & $138 \pm 14$ \\
& + & VUF-K-8788 & $1 \mu \mathrm{M}$ & $154 \pm 14$ \\
& + & & $3 \mu \mathrm{M}$ & $167 \pm 8$ \\
& + & & $10 \mu \mathrm{M}$ & $169 \pm 15$ \\
& + & & $30 \mu \mathrm{M}$ & $155 \pm 13$ \\
& + & Terfenadine & $100 \mu \mathrm{M}$ & $144 \pm 24$ \\
ICAM-1 & + & Dimethylfumarate & $100 \mu \mathrm{M}$ & $92 \pm 2^{* *}$ \\
& - & - & - & $108 \pm 5$ \\
& + & - & - & $308 \pm 15$ \\
& + & VUF-K-8788 & $1 \mu \mathrm{M}$ & $324 \pm 13$ \\
& + & & $3 \mu \mathrm{M}$ & $342 \pm 9$ \\
& + & & $10 \mu \mathrm{M}$ & $334 \pm 37$ \\
& + & & $30 \mu \mathrm{M}$ & $348 \pm 18$ \\
& + & Terfenadine & $100 \mu \mathrm{M}$ & $175 \pm 32^{* *}$ \\
& + & Dimethylfumarate & $100 \mu \mathrm{M}$ & $194 \pm 9^{* *}$ \\
& & &
\end{tabular}

HUVEC were seeded on 6-well plates at $1 \times 10^{5}$ cells in $2 \mathrm{ml}$ of HUVE cell medium (MCDB 104 containing $5 \% \mathrm{FCS}, 5 \mu \mathrm{g} / \mathrm{ml} \mathrm{ECGs} \mathrm{and} 10 \mu \mathrm{g} / \mathrm{ml}$ heparin), and cultured for $3 \mathrm{~d}$. HUVE cell monolayers were rinsed with $1 \mathrm{ml}$ of warmed PBS, and added with TNF- $\alpha(20 \mathrm{ng} / \mathrm{ml})$ and each compound in $2 \mathrm{ml}$ of DMEM/F12 medium containing $10 \%$ FBS and $100 \mu \mathrm{g} / \mathrm{ml}$ endothelial mitogen. After $24 \mathrm{~h}$ incubation, HUVEC were collected with $20 \%$ trypsin-EDTA. VCAM-1 and ICAM-1 expression on HUVEC were analyzed by FACScan. The data are represented as the mean \pm S.D. of three experiments. $* * p<0.01$. MFI: mean fluorescence intensity.

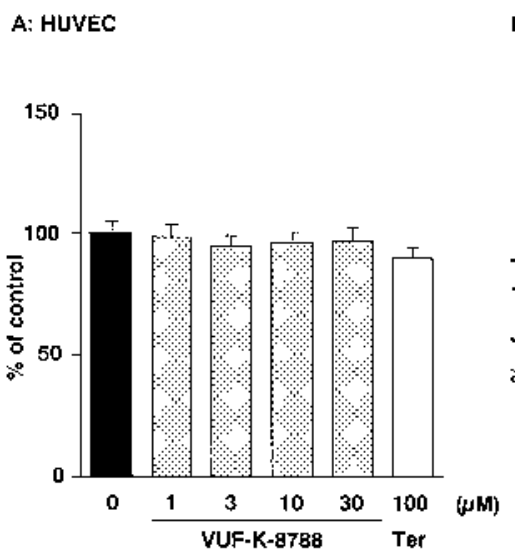

the results reported by Takeda et al.; the LAR was accompanied by eosinophilia, an increase of water content of whole lung, airway hyperresponsiveness and an increase in the amount of histamine, $\mathrm{LTD}_{4}$ and $\mathrm{TXB}_{2}{ }^{8}{ }^{8}$ These results suggested that the histopathological changes were linked with the LAR followed by the airway hyperresponsiveness.

In a previous study, we reported that VUF-K-8788 inhibited antigen-induced IAR and LAR, airway hyperresponsiveness and the infiltration by eosinophils of bronchoalveolar lavage fluids in guinea pigs. ${ }^{10)}$ In the present study, we investigated the effects of VUF-K-8788 on the histopathological changes in the lung parenchyma of guinea pigs during the LAR. VUF-K-8788 inhibited the hyperplasia of airway epithelium, the perivascular edema and the infiltration by eosinophils. The effects of VUF-K-8788 observed in the histological study might contribute to the inhibition of the LAR and the airway hyperresponsiveness. On the contrary, terfenadine did not inhibit the eosinophilia although it inhibited the hyperplasia of airway epithelium and the perivascular edema significantly. In our previous report, terfenadine failed to inhibit the LAR. ${ }^{10)}$ This evidence would indicate the importance of eosinophils to evoke a LAR in the asthmatic model in guinea pigs. In fact, it was reported that the LAR was associated with an increased number of eosinophils in the bronchial mucosa in humans and mice. ${ }^{7,19)}$ Cieslewicz et $a l$. also reported that inhibition of the influx of eosinophils was associated with the inhibition of LAR. ${ }^{19)}$

To study the mechanisms by which VUF-K-8788 suppressed the infiltration by eosinophils, we investigated the effect of VUF-K-8788 on eosinophil-adhesion with HUVEC. VUF-K-8788 inhibited eosinophil-adhesion to HUVEC without a cytotoxic effect at the concentration of $10-30 \mu \mathrm{M}$. Eosinophils adhere to and migrate across the lung microvasculature to exert their effector functions in the lung parenchyma and the airways. This process is regulated by adhesion molecules including VCAM-1 and ICAM-1. ${ }^{20)}$ In our investigation, VUF-K-8788 did not inhibit the expression of VCAM-1 or ICAM-1. It might antagonize the binding of adhesion molecules or inactivate eosinophils to adhere to endothelial cells, although the precise mechanisms were to be investigated.
B:Eosingphlls

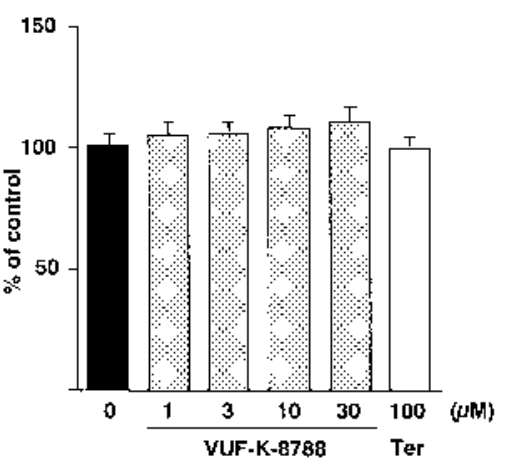

Fig. 6. Cytotoxicity of VUF-K-8788, Terfenadine and Dimethylfumarate for HUVEC and Eosinophils

A: HUVEC were seeded on 96 -well plates at $1 \times 10^{5}$ cells in $100 \mu$ l of HUVE cell medium (D-MEM/F12 medium containing $10 \% \mathrm{FBS}$ and $100 \mu \mathrm{g} / \mathrm{ml}$ bovine endothelial mitogen). After $1 \mathrm{~h}$ incubation, VUF-K-8788, terfenadine or dimethylfumarate was added to confluent HUVEC in $100 \mu \mathrm{l}$ of HUVEC medium. Following $24 \mathrm{~h}$ incubation, cytotoxicity was evaluated by the method of crystal violet. B: Eosinophils were seeded on 96 -well plates at $1 \times 10^{5}$ cells in $100 \mu 1$ of RPMI medium containing $10 \%$ FBS. After $1 \mathrm{~h}$ incubation, VUF-K- 8788 or terfenadine was added to eosinophils in $100 \mu \mathrm{l}$ of HUVE cell medium. Following $24 \mathrm{~h}$ incubation, cytotoxicity was measured by WST-1 assay. Each value represents the mean \pm S.E.M. of 5 experiments. 
It is controversial which is more important, VCAM-1 or ICAM-1, in adhesion of eosinophils to endothelial cells. Akagi described that expression of ICAM-1 participated in the eosinophil recruitment, and that some antihistamines (i.e., azelastine, oxatomide, levocavastine, terfenadine and loratadine) reduce ICAM-1 expression. ${ }^{21)}$ Yamamoto et al. reported that the VCAM-1/ $\alpha 4$ integrin pathway is more important than the ICAM-1/ $\beta 2$ integrin pathway in adhesion of eosinophils to human pulmonary microvascular endothelial cells. $^{22)}$ In the present study, terfenadine did not inhibit the adhesion of eosinophils to HUVEC, although it suppressed the expression of ICAM-1. These results might support the latter hypothesis indirectly. Indeed, several studies on the eosinophilic inflammation in animals, in which blocking monoclonal antibodies were used, have demonstrated important roles of the interaction of VCAM-1 and VLA-4 during the eosinophil recruitment processes. ${ }^{15,23-25)}$

In conclusion, the repeated exposure to OA of guinea pigs induced LAR accompanied by the inflammatory features of hyperplasia of airway epithelial cells, perivascular edema, and infiltration by inflammatory cells, especially eosinophils; VUF-K-8788 inhibited these histopathological phenomena. The mechanisms by which VUF-K-8788 inhibited LAR is at least attributable to suppression of the inflammatory phenomena and eosinophil adhesion to HUVEC in addition to the antihistaminic effect. Therefore, the new antiallergic drug, VUF-K-8788, should be useful in the treatment of asthmatic patients.

\section{REFERENCES}

1) Hargreave F. E., Dolovich J., Robertson D. G., Kerigan A. T., Can. Med. Assoc. J., 110, 415-442 (1974).

2) Charlesworth E. N., Massey W. A., Kagey-Sobotka A., Norman P. S., Lichtenstein L. M., J. Pharmacol. Exp. Ther., 262, 964-970 (1992).

3) Naclerio R. M., Proud D., Togias A. G., Adkinson N. F., Jr., Meyers D. A., Kagey-Sobotka A., Plaut M., Norman P. S., Lichtenstein L. M., N. Engl. J. Med., 313, 65-70 (1985).

4) Pepys J., Hutchcroft B. J., Am. Rev. Respir. Dis., 112, 829-859
(1975).

5) Hargreave F. E., J. Allergy Clin. Immunol., 83, 525-527 (1989).

6) Cartier A., Thomson N. C., Frith P. A., Roberts R., Hargreave F. E., J. Allergy Clin. Immunol., 70, 170-177 (1982).

7) De Monchy J. G. R., Kauffman H. F., Venge P., Am. Rev. Respir. Dis., 131, 373-376 (1985).

8) Takeda H., Kogame A., Tanaka H., Nagai H., Prostaglandins, 54, 805 -820 (1997).

9) Takizawa T., Matsumoto J., Tohma T., Kanke T., Wada Y., Nagao M., Inagaki N., Nagai H., Zhang M. Q., Timmerman H., Jpn. J. Pharmacol., 86, 55-64 (2001).

10) Takizawa T., Yamada T., Takahashi Y., Tanaka H., Wada Y., Nagai H., Pharmacology, 59, 127-134 (1999).

11) Springer T. A., Nature (London), 346, 425-434 (1990).

12) Springer T. A., Cell, 76, 301-314 (1994).

13) Bochner, B. S., Schleimer, R. P., J. Allergy Clin. Immunol., 94, 427438 (1994).

14) Blyth D. I., Pedrick M. S., Savage T. J., Hessel E. M., Fattah D., Am. J. Respir. Cell Mol. Biol., 14, 425-438 (1996).

15) Sagara H., Matsuda H., Wada N., Yagita H., Fukuda T., Okumura K., Makino S., Ra C., Int. Arch. Allergy Immunol., 112, 287-294 (1997).

16) Saito K., Oku T., Ata N., Miyashiro H., Hattori M., Saiki I., Biol. Pharm. Bull., 20, 345-348 (1997).

17) National Heart, Lung and Blood Institute. "Global Initiative for Asthma: Global Strategy for Asthma Management and Prevention," NHLBI/WHO workshop report. NHLBI publication No. 95-3659 (1995).

18) Redington A. E., Howarth P. H., Thorax, 52, 310-312 (1997).

19) Cieslewicz G., Tomkinson A., Adler A., Duez C., Schwarze J., Takeda K., Larson K. A., Lee J. J., Irvin C. G., Gelfand E. W., J. Clin. Invest., 104, 301-308 (1999).

20) Seminario M. C., Gleich G. J., Curr. Opin. Immunol., 6, 860-864 (1994).

21) Akagi M., Folia Pharmacol. Jpn., 111, 217-222 (1998).

22) Yamamoto H., Sedgwick J. B., Busse W. W., J. Immunol., 161, 971977 (1998).

23) Weg V. B., Williams T. J., Lobb R. R., Nourshargh S., J. Exp. Med., 177, 561-566 (1993).

24) Abraham W. M., Sielczak M. W., Ahmed A., Cortes A., Lau-redo I. T., Kim J., Pepinsky B., Benjamin C. D., Leone D. R., Lobb R. R., Weller P. F., J. Clin. Invest., 93, 776-787 (1994).

25) Sriramarao P., DiScipro R. G., Cobb R. R., Cybulsky M., Stachnick G., Castaneda D., Elices M., Broide D. H., Blood, 92, 592-601 (2000). 